\title{
VOCABULARIO EN LENGUA CHIAPANECA DE FINES DEL SIGLO XVIII
}

\author{
Vocabulary of the Chiapaneca Language from the Late 18th Century \\ Carlos Uriel del Carpio-Penagos \\ Juan Ramón Álvarez-Vázquez
}

$\amalg$ n un documento fechado el 9 de octubre de $1787,{ }^{1}$ firmado en el palacio de San Ildefonso, España, por el conde de Floridablanca, secretario del despacho de Estado del rey Carlos III, se ordena a los virreyes de México, Perú, Santa Fe y Buenos Aires, así como al gobernador de Filipinas y al presidente de la Audiencia de Guatemala, que comisionen "a las personas que estimaran conveniente" para que adquieran a la mayor brevedad, y envíen con igual prontitud, un conjunto de obras escritas y la traducción de una lista de palabras, indicando la ortografía para expresarlas, en las diversas lenguas que se hablan en los territorios bajo su jurisdicción. La orden se expidió para complacer una petición efectuada al rey de España por la emperatriz de Rusia, Catalina Segunda La Grande. La lista de obras solicitadas es la siguiente:

1. D. Bergamo, Vocabulario de Panpango en Romance, Manila, 1732, et al.

2. De Noreda y P. de San Lucar, Vocabulario de la lengua Tagala, Manila, 1754, fol.

3. Fr. Pareja, Gramática timuquana, México, 1614.

4. A. de Olmos, Diccionario y gramática de la lengua mexicana.

Carlos Uriel del Carpio Penagos, antropólogo, investigador titular del Centro de Estudios Superiores de México y Centroamérica de la Universidad de Ciencias y Artes de Chiapas, México. Profesor titular de la Universidad Autónoma de Chiapas, México. Correo electrónico: carlitosuriel@hotmail.com.
5. Ph. Ruiz del Corral, Vocabularium pocomanun.

6. A. Ruíz de Montoya, Gramática guayana, Madrid, $1639,8^{\circ}$.

7. Lexicon guayanum, Madrid, 1640.

8. D. Gonzalez Holguín, Gramática quichuana, Lima, 1607,4 .

9. Lexicon quichuanum, Lima, $1608,4^{\circ}$.

10. L. de Valdivia, Grammatica et lexicon milcayac.

11. E. Vega, Gramática et lexicon maromisic.

12. L. Bertonio, Gramática de la lengua aymara, Roma, 1603.

13. L. Figuero, Gramática brasiliana, Luisab.12.

14. D. Collado, Gramática japonensium lingue, Rome. 1632. 4

15. Diccionario japonicum, Rome, 1632. 4.

En hojas aparte, señaladas con el número 2, se indican las palabras a traducir, organizadas en tres columnas y por campos semánticos. Los idiomas hablados en la Audiencia de Guatemala a los que se tradujeron estas palabras fueron el kiche, el cachiquel $^{2}$ o guatemalica y el j,tutuhil o zutugil; en estos idiomas se tradujeron en orden alfabético, mientras que al quiche, cacchí, poconchí, pocoman, pupuluca, ${ }^{3}$ cakchiquel, chol, zotul, tzendal, chanabal, ${ }^{4}$ zoque,

Juan Ramón Álvarez Vázquez, antropólogo, estudiante de la maestría en historia del programa interinstitucional UNICACH-UNACH, México. Correo electrónico: alvarezjuanr@hotmail.com.

Recibido: 17 de marzo de 2013 
subinha o subiná, ${ }^{5}$ chapaneca (sic), mam, cabecara, viceyta, ${ }^{6}$ lean y mulía o tica y terrava ${ }^{7}$ los traductores conservaron en lo posible la organización en columnas y campos semánticos del documento original. Además, estas palabras las tradujeron también al quechua, aymara y tagalo (ver cuadro 1$){ }^{8}$

En total son 444 palabras organizadas en tres columnas y agrupadas por campos semánticos. Avena aparece dos veces, una de ellas escrita abena. En el primer caso se refiere al cereal, pero en el segundo no existe en el diccionario actual de la lengua española aunque es probable que para la época existiera, ya que el traductor del chiapaneca, fray Francisco Montoya, lo incluye en la lista pero indica "no hay", aunque otros traductores, como el que tradujo al zoque, Feliciano Antonio Vivero, da acepciones diferentes para cada palabra (Avena=Yatzi muc; Abena=Yatzi paá).

Por otra parte, en ese tiempo no se respetaban, como hoy día, reglas ortográficas precisas, de manera que una misma palabra se escribía de diversas maneras. Por ejemplo, palabras que hoy se escriben con h inicial como "hogar" u "hoy", en el documento aparecen sin h; o palabras que hoy se escriben con j como "mejilla", entonces se escribían con x (mexilla). Se respetó en todos los casos la ortografía original. También aparece dos veces la palabra vajo, la primera junto a palabras que indican tamaño, como "grande", "pequeño" y "alto"; y la segunda junto a sobre, por lo que es evidente que en este caso equivale a los actuales adverbios de lugar "abajo" o "debajo".

Esta lista fue copiada por algún funcionario encargado de enviarla a los traductores e introdujo algunos cambios con respecto a la original. El primer cambio consistió en organizar los vocablos en cuatro columnas. Además de esto, transformó algunas palabras en otras de grafía parecida, por ejemplo, armar por amar, ocular por ocultar y carrera por carreta, cambios incomprensibles si se toma en cuenta la organización en campos semánticos del original. Por ejemplo, carreta estaba en medio de otras que aludían a actividades agrícolas, como arar, arado, rastrillo y cosecha (líneas 109 a 113, segunda columna); amar estaba junto a amor (líneas 37 y 38, primera columna). Otras se eliminaron, como ysla, mil, piso y rastrillo.
Además, fray Francisco Montoya, el traductor o recopilador de los vocablos chiapanecas correspondientes, fue muy literal, por lo que varias palabras de la lista carecieron de traducción, indicando para las mismas la expresión "No hay" en lugar de buscar un equivalente. Por ejemplo, es inverosímil que no exista una palabra nativa para indicar "guerra" tratándose de una sociedad que, según las fuentes, era eminentemente guerrera; o "liebre", que si no existía en chiapaneca pudo haber tomado la palabra que indicaba "conejo" para traducirla, como lo hizo el traductor del zoque, quien tradujo liebre como caxsla cuía — caxsla o caxlan era la palabra indígena para designar a los españoles y cuíá significa conejo, es decir, tradujo liebre como "conejo de los españoles" - . La palabra vajo, que en la lista original aparece dos veces pero significando cosas diferentes, en ambos casos la tradujo como Cupúmu, lo que nos deja con la impresión de que los chiapanecas no diferenciaban entre "bajo" para referirse al tamaño de una persona y "bajo" como sinónimo de "debajo de". También repitió dos veces la palabra miel —nambápu-, y es probable que esto se deba a que el mil de la lista original se convirtió en una segunda miel, lo cual explicaría su supresión de la lista original.

\section{Lengua chiapaneca}

El chiapaneca es una lengua extinta perteneciente a un grupo lingüístico denominado oto-mangue, que incluye diversos idiomas hablados en los estados de Oaxaca y Guerrero, como el popoluca, el chatino y el tlapaneca, entre otros. Los hablantes originales provenían del centro de México, concretamente de la región de Cholula, quienes emigraron hacia el sur durante los siglos VII al IX d.C. (Borden, s.f.: 1; Kinloch, 2008: 19).

Un segmento de los grupos migrantes se estableció en la depresión central de Chiapas, en las riberas del río Grijalva, dando origen al pueblo chiapaneca, y otro continuó su camino hacia el sur por la costa del Pacífico hasta establecerse en un territorio comprendido desde el Golfo de Fonseca, donde fueron conocidos como chorotegas o cholutecas, hasta Orotina, en el Golfo de 
Nicoya, en la actual frontera entre Nicaragua y Costa Rica, donde se les conoció como mangues (Arrechavala, 2009: 32). Tanto el chiapaneca como el mangue se extinguieron a fines del siglo XIX.

Existen diversos documentos coloniales publicados en lengua chiapaneca, el más importante de los cuales es el Arte de la lengua chiapaneca compuesto por el padre Fray Juan de Albornoz de la orden de predicadores de la provincia de san Vicente de Chiapa y Guatemala, de fines del siglo XVII. Otros son el publicado por Mario Humberto Ruz y Claudia Margarita Báez en el año 2003, Las lenguas del Chiapas colonial, donde incluyen el texto de Albornoz; un texto de autor anónimo denominado Ordenanzas de la cofradía de la Vera Cruz; Sermones y algunas cosas curiosas en lengua chiapaneca, de Joan Nuñez, escrito en la segunda mitad del siglo XVII; otro documento de autor anónimo denominado Tabla de los evangelios que se cantan en la iglesia todos los domingos, días de la cuaresma y fiestas principales de todo el año, probablemente también de mediados del siglo XVII; y la Doctrina en lengua chiapaneca, de Luis Barrientos. Estas fuentes permiten estudiar aspectos fonológicos, morfológicos y sintácticos del idioma chiapaneca del siglo XVII.

Posteriormente, la cultura chiapaneca comenzó un proceso de integración a la cultura española que condujo a la extinción del idioma, sobreviviendo sin embargo una extensa toponimia, así como nombres patronímicos y algunos rituales. En 1883, el obispo de Chiapas, Moreno y Castañeda, informa en una carta a Francisco Pimentel, autor del Cuadro descriptivo y comparativo de las lenguas de México, que "el chiapaneco... es un idioma muerto, enteramente perdido, pues la tribu que lo hablaba, mezclada entre los ladinos, como aquí los llaman, habla el español" (Pimentel, 1883: 308, citado por Borden, s.f.: 5). Sin embargo, un reciente estudio de historia demográfica sobre el área chiapaneca argumenta que el idioma no se extinguió por integración de sus hablantes a la cultura española, sino porque fueron diezmados por epidemias y hambrunas y los sobrevivientes tuvieron que emigrar a otras regiones. Dichas epidemias y hambrunas se presentaron precisamente en el curso del siglo XVIII (Obara Saeki, 2010).
El alemán Karl Hermann Berendt publicó a fines del siglo XIX los documentos originales El libro de cuentas de la cofradía del Rosarioen el pueblo de Suchiapa (1796-1821), Apuntes y estudios sobre la lengua chapaneca (1869-1870), Pasión del Jueves Santo (1818) y Pasión en lengua chiapaneca, canciones de los indios de Suchiapa (1870). Estos documentos constituyen valiosas fuentes para el estudio de la lengua y la cultura chiapaneca en sus estertores y se complementan con el del filólogo francés Lucien Adam publicado en 1887, titulado La langue chiapaneque: observations grammaticales, vocabulaire méthodique, textes inédites, textes rétablies.

En el siglo XX, los trabajos sobre la lengua y la cultura chiapaneca más importantes, sin duda, son: los de Navarrete, The Chiapanec, History and Culture (1966), que es sobre todo un estudio de la arqueología cerámica y de los restos de edificios prehispánicos; el de Mario Aguilar Penagos, Diccionario y gramática de la lengua chiapaneca (2011), en dos tomos; el Prontuario de la lengua chiapaneca, de Mario Nandayapa, que es un estudio detallado de la gramática, cuya intención es facilitar la traducción de documentos del español al chiapaneca (Nandayapa, 2012); y el ya citado trabajo de Tadashi Obara Saeki, denominado Ladinización sin mestizaje. Historia demográfica del área chiapaneca 1748-1813.

El vocabulario que damos a conocer se elaboró en Chiapa - hoy Chiapa de Corzo - y abona al conocimiento de la lengua chiapaneca del siglo XVIII, época para la cual no existían, hasta hoy, documentos conocidos, como vimos en el resumen anterior sobre los principales trabajos publicados en y sobre esta lengua. Un aporte importante del presente vocabulario es la traducción de los números del 1 al ll, así como de las palabras para designar 20, 30 y 100, ya que el diccionario de Mario Aguilar Penagos solamente incluye el uno - tiche- Se conserva el orden de las palabras del documento original manuscrito, la organización en columnas y se le agregó la primera columna para facilitar el conteo de las palabras. Cada cuadro del 2 al 11 representa una hoja manuscrita en el documento original (ver cuadros 2 al 11 ).

El traductor, fray Francisco Montoya, incluyó las siguientes aclaraciones en su informe al gobernador intendente Francisco Saavedra y Carvajal: 
Para inteligencia de la traducción pongo las advertencias siguientes:

La silaba cha se debe escrivir con q. en lugar de c. pero lla los yndios casi pronuncian dicha dicción con en castilla.

Antes de B, D, Y, se debe poner N, assi en el principio como en el medio de las dicciones y se pronuncia con suavidad como decir en castellano el termino pendiente dividido de esta manera: pe-ndiente. Otro. Ambiente, A-nbiente.

La letra $\mathrm{H}$, tiene fuerza de J, assi al principio de el termino, como en el medio; y ponerse h, es porque la mitad de la fuerza se arroja por la voca y la otra por las narizes.

Las rallitas o asentos que pongo sobre de las vocales es para que se conosca en donde cae la fuerza de la pronunciación; y sí en un termino se ponen dos, es porque ambas son largas y con todo eso, esta lengua es mui violenta en su pronunciación.

Señor Gobernador Yntendente Don Francisco Saavedra y Carvajal, Señor Governador Yntendente= Mi mui estimado Dueño y Señor= remito a Usted el diccionario puesto en lengua Chapaneca sus correspondientes términos expresivos de sus significados hecho con la maior prolixidad que mi inutilidad permite, y se acompaña un papel de mediana orthographia para su inteligencia y V. S perdonará los defectos=

Quedo para servir a V. S. y rogamos a Dios Nuestro Señor guarde su vida muchos años= Chiapa y febrero veinte $\mathrm{y}$ siete de mil setecientos ochenta y nueve= Humilde servidor de V.S, L. M. D. V.S. Fray Francisco Montoya.

El gobernador intendente a su vez envía el documento al presidente de la Audiencia de Guatemala, Josef de Estachería:

Mui Ylustre señor Presidente Don Josef Estachería= Mui Ylustre Señor: a consequencia del superior despacho de V. S. de veinte y nueve de diciembre anterior se han traducido las voces que expresa la lista No. 2 en los varios idiomas que hablan los yndios en los Partidos de esta Yntendencia por los sujetos mas instruidos e inteligentes en ellos, con las traducciones originales acompaño a V. S. en el referido despacho, no haviendose podido recoger libro alguno de los que señala la lista No. 1 ni otro que sea conducente al asunto. Nuestro Señor guarde a V. S muchos años, Ciudad Real, marzo 3 de mil setecientos ochenta y nueve. Mui ylustre señor Francisco Saavedra.

Real Palacio catorce de marzo de mil setecientos ochenta y nueve. Pongase con los antecedentes y al señor Oydor Fiscal en estado. Rubricado del Mui Ylustre Señor Presidente Governador y Capitan General de este Reyno. Ygnacio Guerra y Marchán. ${ }^{9}$

\section{Notas}

${ }^{1}$ AGI, Documentos Escogidos 1, No. 165, doc. $2{ }^{2}$.

${ }^{2}$ El cachiquel y el cakchiquel se presentan como dos lenguas distintas en el documento, aunque la Academia de Lenguas Mayas de Guatemala indica que hoy día existe el kaqchiquel con esta ortografía. Lo mismo ocurre con el quiché o kiche - cuya ortografía correcta es k'iche'-, que en el documento en cuestión se presenta como dos lenguas diferentes.

${ }^{3}$ Pupuluca o Popoluca en náhuatl significa extranjero. En el siglo XVIII había por lo menos dos idiomas en la Audiencia de Guatemala a los que se denominaba de esta manera. Uno era el que se hablaba en San Andrés Cuilco, una zona muy montañosa en las cercanías de Motozintla. A fines del siglo XVIII, fray Ramón de Pineda y fray Miguel Hermenegildo Muñoz dicen lo siguiente sobre el idioma de Cuilco: "aquí se dice popoluca, es particular de esta parroquia, mezclado del mam y chiapaneco, y aun los del pueblo de Motozintla lo hablan diferente que los otros, de modo que no entienden ni aun son entendidos de los otros pueblos del curato" (Cortés, 1958: II, 138, citado por De Solano), por lo que podría tratarse del mochó o motocintleco. El popoluca-xinca es el otro, el cual se hablaba en el sur de Guatemala, en la zona de frontera con El Salvador. El xinca es un idioma del grupo tseltal.

${ }^{4}$ Es probable que chanabal designara al actualmente conocido como tojolabal. 
5 Kauffman, que revisó el vocabulario que aquí comentamos, indica que la traducción al subinha está compuesta de palabras tseltales, tojolabales y chujes (Kauffman, 1974, citado por Lenkersdorf, 1982: 73). Francisco Fernández y González al parecer también se basó en el vocabulario comentado, ya que en su obra Los lenguajes hablados por los indígenas del norte y el centro de América, dada a conocer originalmente como conferencia el 29 de febrero de 1892 y publicada en forma impresa en Madrid en 1893, proporciona los pronombres personales y la conjugación del verbo ser, así como los números del 1 al ll, 20, 30 y 100. No cita su fuente pero evidentemente proviene del vocabulario. Hace referencia al subinha, indicando los nombres de los números del 1 al ll, como aparecen en el vocabulario. El documento original indica que el vocabulario fue traducido al subinha en Zocoltenango por el capellán Joseph Anselmo Ortis (AGI, Documentos Escogidos 1_Nl65_40_0204). En Socoltenango en esa época se hablaba tseltal.

${ }^{6}$ Se trata del bribri, idioma de Costa Rica, con una población aproximada de 12000 hablantes en el año 2000.

${ }^{7}$ Térraba, tiribí o téribe es un idioma hablado hoy día en el noroeste de Panamá y en el sur de Costa Rica. Es de la familia chibcha.

${ }^{8}$ El material lingüístico en su totalidad fue publicado en 1882 en el Boletín de la Sociedad Geográfica de Madrid, pero es un material difícil de conseguir, por lo que es prácticamente desconocido (ver books.google.com.mx/ books?id=tSQFAAAAQAAJ).

${ }^{9}$ Ygnacio Guerra y Marchán era el escribano.

\section{Referencias bibliográficas}

Aguilar Penagos, Mario (2012), Gramática de la lengua chiapaneca, México: FONCA-CONACULTA, 2 t.
Arrechavala Romero, Jilma (coord.) (2009), Nuestras comunidades. Una mirada histórica de los pueblos indígenas del Pacífico, Centro y Norte de Nicaragua, Managua: Universidad Nacional Autónoma de Nicaragua.

Borden Eng, Rubén(s.f.), "Fuentesy estudios sobrelaslenguas del grupo chiapaneco-mangue”, México: UNAM. ‘http:// www.academia.edu/1693369/Fuentes_y_estudios_sobre las_lenguas_del_grupo_chiapaneco-mangue> $\quad[29$ de marzo de 2013].

De Solano, Francisco (1969), “Áreas lingüísticas y población de habla indígena de Guatemala en 1772", en Revista Española de Antropología Americana, vol. IV, pp. 145-200, 〈http://revistas.ucm.es/index.php/REAA/article/view/ REAA6969110145A/25552> [2 de abril de 2013].

Francisco Fernández y González (1893), Los lenguajes hablados por los indígenas del norte y el centro de América, Madrid: Establecimiento Tipográfico Sucesores de Rivadeneyra, Impresores de la Real Casa.

Kinloch Tijerino, Frances (2008), Historia de Nicaragua, Nicaragua: IHNCA-UCA.

Lenkersdorf, Gudrum (1982), "Contribuciones a la historia colonial de los tojolabales", en Mario Humberto Ruz (ed.), Los legítimos hombres. Aproximación antropológica al grupo tojolabal, vol. IV, México: UNAM.

Nandayapa, Mario (2012), Prontuario de la lengua chiapaneca, México: FONCA-CONACULTA.

Navarrete, Carlos (1966), The Chiapanec, History and Culture, Papers of The New World Archaeological Foundation, núm. 16, Provo, Utah: Brigham Young University.

Obara-Saeki, Tadashi (2010), Ladinización sin mestizaje Historia demográfica del área chiapaneca 1748-1813, Tuxtla Gutiérrez, Chiapas: Consejo Estatal para la Cultura y las Artes de Chiapas.

Ruz, Mario Humberto y Claudia Margarita Báez (eds.) (2003), Las lenguas del Chiapas colonial. Manuscritos. Volumen tres: lengua chiapaneca, México: UNAM. 
TRANSCRIPCIÓN DEL DOCUMENTO

Cuadro 1. Lista de palabras cuya traducción solicita la emperatriz de Rusia Catalina Segunda

\begin{tabular}{|c|c|c|c|}
\hline & 1 & 2 & 3 \\
\hline 1 & Dios & Boca & Hueso \\
\hline 2 & Padre & Garganta & Oido \\
\hline 3 & Madre & Labios & Vista \\
\hline 4 & Hijo & Dientes & Ver \\
\hline 5 & Hija & Lengua & Gusto \\
\hline 6 & Hermano & Barba & Olfato \\
\hline 7 & Hermana & Cuello & Oler \\
\hline 8 & Marido & Hombro & Tacto \\
\hline 9 & Muger & Codo & Voz \\
\hline 10 & Doncella & Mano & Hablar \\
\hline 11 & Mozo & Brazo & Razonamiento \\
\hline 12 & Niño & Dedos & Palabra \\
\hline 13 & Hombre & Uñas & Nombre \\
\hline 14 & Gentes & Pecho & Gritar \\
\hline 15 & Caveza & Vientre & Grito \\
\hline 16 & Cara & Espalda & Ruido \\
\hline 17 & Nariz & Pie & Ahullido \\
\hline 18 & Narizes & Rodilla & Llorar \\
\hline 19 & Ojo & Corazón & Reir \\
\hline 20 & Cejas & Estomago & Cantar \\
\hline 21 & Pestañas & Sangre & Estornudar \\
\hline 22 & Oreja & Leche & Temblar \\
\hline 23 & Frente & Piel & Suspirar \\
\hline 24 & Cavellos & Carne & Bostezar \\
\hline 25 & Mexillas & Tu eres & Silvar \\
\hline 26 & Echarse & El es & Parir \\
\hline 27 & Para (tu) & Nosotros somos & Familia \\
\hline 28 & Ir & Vosotros sois & Matrimonio \\
\hline 29 & Vé (tu) & Aquellos son & Nupcias \\
\hline 30 & Véte & Fué & Viuda \\
\hline 31 & Dormir & Comer & Vivir \\
\hline 32 & Sueño & Yo como & Vida \\
\hline 33 & Saltar & Tu comes & Cuerpo \\
\hline 34 & Tener & Aquel come & Estatura \\
\hline 35 & Correr & Beber & Espíritu \\
\hline 36 & Baylar & Nutrir & Alma \\
\hline
\end{tabular}




\begin{tabular}{|c|c|c|c|}
\hline 37 & Amar & Tomar & Morir \\
\hline 38 & Amor & Sacudir (golpear) & Muerte \\
\hline 39 & Gozoso & Llevar & Viejo \\
\hline 40 & Gozo & Echar & Joven \\
\hline 41 & Tristeza & Desgarrar & Grande \\
\hline 42 & Dolor & Verter & Pequeño \\
\hline 43 & Pena & Dar & Alto \\
\hline 44 & Trabajo & $\mathrm{Da}$ (da tu) & Vajo \\
\hline 45 & Perezoso & Cortar & Frio \\
\hline 46 & Yo & Ocultar & Caliente \\
\hline 47 & Tu & Fuerza & Ardiente \\
\hline 48 & Aquel & Facultad & Sano \\
\hline 49 & Nosotros & Poder & Bien (adv.) \\
\hline 50 & Vosotros & Amarillo & Bueno \\
\hline 51 & Aquellos & Azul & Yelo \\
\hline 52 & Ser & Sol & Fuego \\
\hline 53 & Yo soy & Luna & Lumbre \\
\hline 54 & Dichoso & Estrella & Sombra \\
\hline 55 & Malvado & Cielo & Sombrio \\
\hline 56 & Mal & Niebla & Dia \\
\hline 57 & Estulto & Nuve & Noche \\
\hline 58 & Capaz & Arco iris & Mañana \\
\hline 59 & Hermoso & Rayo & Tarde \\
\hline 60 & Agudo & Ayre & Oriente \\
\hline 61 & Redondo & Viento & Occidente \\
\hline 62 & Circulo & Ventilar & Septentrion \\
\hline 63 & Bola & Turbonada & Medio dia \\
\hline 64 & Ligero & Uracan & Verano \\
\hline 65 & Pesado & Vapor & Ynvierno \\
\hline 66 & Duro & Lluvia & Otoño \\
\hline 67 & Fuerte & Rocio & Primavera \\
\hline 68 & Endeble & Granizo & Ora \\
\hline 69 & Delgado & Trueno & Semana \\
\hline 70 & Grueso & Relampago & Año \\
\hline 71 & Ancho & Nieve & Tiempo \\
\hline 72 & Presto & Frio & Mundo \\
\hline 73 & Lento & Elada & Tierra \\
\hline 74 & Blanco & Estaño & Agua \\
\hline 75 & Negro & Plomo & Encina \\
\hline
\end{tabular}




\begin{tabular}{|c|c|c|c|}
\hline 76 & Encarnado & Sal & Viña \\
\hline 77 & Verde & Cal & Llanura \\
\hline 78 & Mar & Veneno & Campo \\
\hline 79 & Rio & Maravilla & Sembrar \\
\hline 80 & Lago & Verdura & Trigo (en general) \\
\hline 81 & Olas & Yerva & Centeno \\
\hline 82 & Ysla & Árbol & Trigo escogido \\
\hline 83 & Arena & Leña & Avena \\
\hline 84 & Arcilla & Bosque & Abena \\
\hline 85 & Polvo & Palo & Cevada \\
\hline 86 & Cieno & Raíz & Guisantes \\
\hline 87 & Montaña & Tronco & Animal \\
\hline 88 & Rivera & Corteza & Pescado \\
\hline 89 & Colina & Rama & Ballena \\
\hline 90 & Valle & Oja & Cangrejo \\
\hline 91 & Profundidad & Flor & Serpiente \\
\hline 92 & Altura & Fruto & Rana \\
\hline 93 & Anchura & Semilla & Gusano \\
\hline 94 & Longitud & Baya /fruta/ & Mosca \\
\hline 95 & Agujero & Cevolla & Mosquito \\
\hline 96 & Fosa & Nuez & Ormiga \\
\hline 97 & Foso & Manzana & Araña \\
\hline 98 & Cueva & Pichón & Aveja \\
\hline 99 & Piedra & Águila & Villa \\
\hline 100 & Oro & Cuervo & Seguir \\
\hline 101 & Plata & Codorniz & Medida \\
\hline 102 & Cobre & Pájaro & Cuchillo \\
\hline 103 & Hierro & Gorrión & Caldero \\
\hline 104 & Miel & Golondrina & Mesa \\
\hline 105 & Buey & Pluma & Banco \\
\hline 106 & Baca & Huevo & Cuba \\
\hline 107 & Ternera & Nido & Navio \\
\hline 108 & Oveja & Pastor & Esquife \\
\hline 109 & Carnero & Arar & Coche \\
\hline 110 & Cabra & Arado & Acarrear \\
\hline 111 & Cuerno & Carreta & Ir en coche \\
\hline 112 & Cavallo & Rastrillo & Construir \\
\hline 113 & Camello & Cosecha & Vestido \\
\hline 114 & Asno & Limite & Pelliza \\
\hline
\end{tabular}




\begin{tabular}{|c|c|c|c|}
\hline 115 & Cerdo & Casa & Media \\
\hline 116 & Perro & Choza & Zapato \\
\hline 117 & Gato & Tienda & Gorro \\
\hline 118 & León & Puerta & Faja \\
\hline 119 & Oso & Ogar & Seda \\
\hline 120 & Lobo & Piso & Lana \\
\hline 121 & Zorra & Ciudad & Algodón \\
\hline 122 & Liebre & Guardia & Lino \\
\hline 123 & Raton & Ley & Quien \\
\hline 124 & Gallo & Yugo & Con quien \\
\hline 125 & Gallina & Leer & Con qué \\
\hline 126 & Ganso & Escrivir & Vajo \\
\hline 127 & Pato & Principio & Sobre \\
\hline 128 & Comida & Fin & Sin \\
\hline 129 & Crudo & $\mathrm{Si}$ & En \\
\hline 130 & Cozer & No & Numero \\
\hline 131 & Zerveza & Aora & Uno \\
\hline 132 & Vino & Antes & Dos \\
\hline 133 & Azeyte & Después & Tres \\
\hline 134 & Manteca & Cerca & Quatro \\
\hline 135 & Pan & Lejos & Cinco \\
\hline 136 & Dinero & Aquí & Seis \\
\hline 137 & Ladron & Allá & Siete \\
\hline 138 & Guerra & Ayer & Ocho \\
\hline 139 & Soldado & Oy & Nueve \\
\hline 140 & Contienda & Mañana & Diez \\
\hline 141 & Batería & Hé aquí & Once \\
\hline 142 & Arnes & Ó (particip.) & Veinte \\
\hline 143 & Casco & Como & Treinta \\
\hline 144 & Escudo & Donde & Ciento \\
\hline 145 & Lanza & Quando & Mil \\
\hline 146 & Miseria & Qué & Señor \\
\hline 147 & Victoria & Enemigo & Siervo \\
\hline 148 & Amigo & Príncipe & Rey \\
\hline
\end{tabular}

Fuente: AGI, Documentos Escogidos, 1, núm. 165, doc. 2b. 
Cuadro 2. Vocabulario castellano-chiapaneca, 1789. Hoja 0205

\begin{tabular}{|c|c|c|c|c|}
\hline & Castellano & Chapaneca (sic) & Castellano & Chapaneca (sic) \\
\hline 1 & Dios & Nunbui, I, nDios & Hombro & Ngulúá \\
\hline 2 & Padre & Ýuguá & Codo & Nyíhmí \\
\hline 3 & Madre & Numa & Mano & Ndiya \\
\hline 4 & Hijo & Ñsana & Brazo & Ngúlua, I, nu yindila \\
\hline 5 & Hija & Ñsana & Dedos & Nbañandiya \\
\hline 6 & Hermano, & Mánguhu & Uñas & Manducu \\
\hline 7 & Hermana & Mánguhu & Pecho & Ngupilu \\
\hline 8 & Marido & Nuhí & Vientre & Ndacá \\
\hline 9 & Muger & Nafí & Leche & Nambatú \\
\hline 10 & Doncella & Nacue yafí, yafiquí & Piel & Ndu \\
\hline 11 & Moso & Nacue ýuguá & Carne & Nampúi \\
\hline 12 & Niño & Niño, I, Nacué & Hueso & Nuyí \\
\hline 13 & Hombre & Nuguá & Oydo & Nungupuhúmu \\
\hline 14 & Gentes & Ndípahu & Vista & Numucupamu \\
\hline 15 & Caveza & Nuchamá & Ver & Uñú \\
\hline 16 & Cara & Níu & Gusto & Nuchipaimu \\
\hline 17 & Naríz & Nguíú & Olfato & Nucheimu \\
\hline 18 & Cabellos & Nimbi & Oler & Achí \\
\hline 19 & Mexillas & Nyipá & Tacto & Nutu miñamu \\
\hline 20 & Boca & Yndú & Voz & Nampáyí \\
\hline 21 & Garganta & Ngurí & Hablar & Nbapamó \\
\hline 22 & Lavios & Nyíma indu & Razonamiento & Numbace nálapu \\
\hline 23 & Dientes & Níhi & Palabra & Númbace \\
\hline 24 & Lengua & Nbélu & Nombre & Nui \\
\hline 25 & Barba & Ngu pállí & Gritar & Tahmáme \\
\hline 26 & Cuello & Ngurí & Estornudar & Mangarisehé \\
\hline 27 & Temblar & Ypumamú & Vosotros & Simeému \\
\hline 28 & Suspirar & Nbúna nánbue & Aquellos & Simine \\
\hline 29 & Bostezar & Nbulaní & Ser & $\begin{array}{l}\text { Slimuhumu. } \\
\text { Propiedad }\end{array}$ \\
\hline 30 & Silvar & Umbilima & Yo soy & Simaña \\
\hline 31 & Echarse & Nguputí & Tu eres & Simuña \\
\hline 32 & Para (tu) & Pusá (símu) & Él es & Sumulu ña \\
\hline 33 & $\mathrm{Yr}$ & Yxa & Nosotros somos & Sih mimuña \\
\hline
\end{tabular}

Fuente: AGI, Documentos Escogidos 1_N165_40_0204 y 40_0205. 
Cuadro 3. Vocabulario castellano-chiapaneca, 1789. Hoja 0206

\begin{tabular}{|c|c|c|c|c|}
\hline & Castellano & Chapaneca (sic) & Castellano & Chapaneca (sic) \\
\hline 1 & Ve tú & Pía simu & Vosotros sois & Sime emuna \\
\hline 2 & Vete & Pía simu & Aquellos son & Simé nena \\
\hline 3 & Dormir & Umbí & Fue & No hay \\
\hline 4 & Sueño & Numucumu & Comer & Uta \\
\hline 5 & Saltar & Nbarití & Espalda & Nguhí \\
\hline 6 & Tener & Mahe & Pie & Lácu \\
\hline 7 & Correr & Nbujumú & Rodilla & Nambahmí \\
\hline 8 & Bailar & Aha & Corazón & Nambue \\
\hline 9 & Armar & Lupucucahu & Estómago & Niunambué \\
\hline 10 & Narizes & Nyúí & Sangre & Nihú \\
\hline 11 & Ojo & Nate & Echar & Nbaá \\
\hline 12 & Cejas & Nimbinaté & Desgarrar & Utelumehé \\
\hline 13 & Pestañas & Nimbi múnaté & Verter & Arímá \\
\hline 14 & Oreja & Níha & Dar & Uguau \\
\hline 15 & Frente & Nguré & $\mathrm{Da}$ (da tu) & Uguau (simu) \\
\hline 16 & Trabajo & Nambiamu & Cortar & Umbarita \\
\hline 17 & Perezoso & Narimé & Ocular & Umbacía \\
\hline 18 & Yo & Sime & Fuerza & Nacimahí \\
\hline 19 & Tú & Simu & Facultad & Numucícame \\
\hline 20 & Aquel da & Súne tilapa & Poder & Numucícame \\
\hline 21 & Nosotros & Sínmimu & Parir & Urime \\
\hline
\end{tabular}

Fuente: AGI, Documentos Escogidos 1_N165_40_0206. 
Cuadro 4. Vocabulario castellano-chiapaneca, 1789. Hoja 0207

\begin{tabular}{|c|c|c|c|c|}
\hline & Castellano & Chapaneca (sic) & Castellano & Chapaneca (sic) \\
\hline 1 & Familia & Nimáhí & Agudo & Ambumé \\
\hline 2 & Matrimonio & Numupah miyá lapu & Amor & Nacciymu \\
\hline 3 & Nupcias & Numupah miyá lapu & Gozoso & Namburémé \\
\hline 4 & Viuda & Nafinambavíhúmu & Gozo & Nambanéymu \\
\hline 5 & Vivir & $\mathrm{Hu}$ & Tristeza & Naricuimu \\
\hline 6 & Vida & Numumbucahámu & Dolor & Naýpa \\
\hline 7 & Grito & Numutahmamé & Pena & Nanyitaméymu \\
\hline 8 & Ruido & Nupihmi. I. ruido & Delgado & Naanibatí \\
\hline 9 & Aullido & Ytahmame & Grueso & Cicumé \\
\hline 10 & Llorar & Ynda & Ancho & Nacalumé \\
\hline 11 & Reir & Nbucúmu & Presto & Catetalú \\
\hline 12 & Cantar & Undamú & Lento & Nbuta mulú \\
\hline 13 & Grande & Naripe & Blanco & Andilí \\
\hline 14 & Pequeño & Natacucé & Negro & Nandúha \\
\hline 15 & Alto & Apamé & Encarnado & Nandiú \\
\hline 16 & Vajo & Cupúmu & Verde & Andípa \\
\hline 17 & Frío & Nalamé & Amarillo & Nandicu \\
\hline 18 & Caliente & Natúhmu & Azul & $\begin{array}{l}\text { Nandipa.l. } \\
\text { nyílunimbu }\end{array}$ \\
\hline 19 & Ardiente & Ysimé & Sol & Mapihú \\
\hline 20 & Sano & Apaýme & Luna & Llumú \\
\hline 21 & Bien (adv) & Angame & Estrella & Nafití \\
\hline
\end{tabular}

Fuente: AGI, Documentos Escogidos 1_N165_40_0207. 
Cuadro 5. Vocabulario castellano-chiapaneca, 1789. Hoja 0208

\begin{tabular}{|c|c|c|c|c|}
\hline & Castellano & Chapaneca (sic) & Castellano & Chapaneca (sic) \\
\hline 1 & Bueno & Angame & Cielo & Nacúpahu \\
\hline 2 & Dichoso & Panécaté & Niebla & Nambumé \\
\hline 3 & Malvado & Fangamí & Nuve & Námarí \\
\hline 4 & Mal & Nitángame & Yo como & Síme íta \\
\hline 5 & Estulto & Ylupunumbamé & Tú comes & Simu íta \\
\hline 6 & Capaz & Numbúmumúluhú & Aquel come & Súne íta \\
\hline 7 & Hermoso & Charícame & Beber & Urí \\
\hline 8 & Nutrir & Ypuchí & Tierra & Nacapú \\
\hline 9 & Tomar & Ahe & Agua & Nimbu \\
\hline 10 & Sacudir (golpear) & Nbusamahí & Mar & Numbucupáhu \\
\hline 11 & Llevar & Ypamahé & Río & Néhu \\
\hline 12 & Llubia & Nambahamú & Lago & Nambucámu \\
\hline 13 & Rosio & Nalamé & Olas & Narehumé \\
\hline 14 & Granizo & Nicatuy & Redondo & Alucime \\
\hline 15 & Trueno & Ycuitarimé & Círculo & Nambehé \\
\hline 16 & Relámpago & Ylupíu & Bola & Alucime \\
\hline 17 & Nieve & Numbúménalamé & Ligero & Llarimimulu \\
\hline 18 & Frío & Nalamé & Pesado & Narimé \\
\hline 19 & Elada & Nalamé & Duro & Atápa \\
\hline 20 & Yelo & Nalamé & Fuerte & Atapamé \\
\hline 21 & Fuego & Niú & Endeble & Yteilípuca \\
\hline
\end{tabular}

Fuente: AGI, Documentos Escogidos 1_N165_40_0208. 
Cuadro 6. Vocabulario castellano-chiapaneca, 1789. Hoja 0209

\begin{tabular}{|c|c|c|c|c|}
\hline & Castellano & Chapaneca (sic) & Castellano & Chapaneca (sic) \\
\hline 1 & Lumbre & Niú & Fosa & No hay \\
\hline 2 & Cuerpo & Ndípahúmu & Fozo & No hay \\
\hline 3 & Estatura & No hay & Cueva & Nacúhá \\
\hline 4 & Espíritu & Ánima & Piedra & Núca \\
\hline 5 & Alma & Anima & Oro & $\begin{array}{l}\text { Lo mismo en } \\
\text { lengua }\end{array}$ \\
\hline 6 & Morir & Ybíhí & Plata & $\begin{array}{l}\text { Lo mismo en } \\
\text { lengua }\end{array}$ \\
\hline 7 & Muerte & Numufímu & Cobre & $\begin{array}{l}\text { Lo mismo en } \\
\text { lengua }\end{array}$ \\
\hline 8 & Viejo & Náha & Hierro & Nambucullá \\
\hline 9 & Joven & Nacué lluguá & Estaño & Lo mismo \\
\hline 10 & Hora & Catétalu & Plomo & Lo mismo \\
\hline 11 & Semana & Lo mismo en lengua & Cal & Nambú \\
\hline 12 & Año & Nbutimu & Arcoiris & Nambalutí \\
\hline 13 & Tiempo & Mindamu. I. tiempo & Rayo & Niunucutému \\
\hline 14 & Mundo & Marimulú nacapú & Ayre & Tehú \\
\hline 15 & Viento & Marimulú nacapú & Primavera & Muicahá nbutimú \\
\hline 16 & Ventilar & $\begin{array}{l}\text { Lambatau. I, } \\
\text { chasarali }\end{array}$ & Gusano & Númbu \\
\hline 17 & Turbonada & Latihú & Mosca & Nambamu \\
\hline 18 & Uracán & Tehuyacumé & Mosquito & Nuú \\
\hline 19 & Vapor & Lasúmé & Ormiga & Nahú \\
\hline 20 & Flor & Núlu & Araña & Nasiátila \\
\hline 21 & Fruto & Nyimulámu & Abeja & Numbú nhapu \\
\hline
\end{tabular}

Fuente: AGI, Documentos Escogidos 1_N165_40_0209. 
Cuadro 7. Vocabulario castellano-chiapaneca, 1789. Hoja 0210

\begin{tabular}{|l|l|l|l|l||}
\hline & \multicolumn{1}{|c|}{ Castellano } & \multicolumn{1}{|c|}{ Chapaneca (sic) } & \multicolumn{1}{c|}{ Castellano } & Chapaneca (sic) \\
\hline 1 & Semilla & Namúmu & Miel & Nambápu \\
\hline 2 & Baya (fruta) & No hay & Buey & Nbúllucí \\
\hline 3 & Sebolla & Nambuchá & Baca & Lo mismo en \\
lengua
\end{tabular}

Fuente: AGI, Documentos Escogidos 1_N165_40_0210. 
Cuadro 8. Vocabulario castellano-chiapaneca, 1789. Hoja 0211

\begin{tabular}{|c|c|c|c|c|}
\hline & Castellano & Chapaneca (sic) & Castellano & Chapaneca (sic) \\
\hline 1 & Pichón & Nbaña nampuhú & Trigo escogido & $\begin{array}{l}\text { Nama catíla } \\
\text { nuhecamu }\end{array}$ \\
\hline 2 & Aguila & Náguagua & Avena & No hay \\
\hline 3 & Cuerbo & Náhua & Abena & No hay \\
\hline 4 & Codorníz & Cuychí & Sebada & $\begin{array}{l}\text { Lo mismo en } \\
\text { lengua }\end{array}$ \\
\hline 5 & Veneno & Numbafiimu & Guisante & Ticica namutá \\
\hline 6 & Maravilla & Niluturípihá. flor & Animal & Ndípahuchiá \\
\hline 7 & Verdura & Níhu & Pescado & Nangací \\
\hline 8 & Yerva & Nímu & Ballena & $\begin{array}{l}\text { Nangacínambana } \\
\text { acumé }\end{array}$ \\
\hline 9 & Árbol & Niyá & Cangrejo & Nacutí \\
\hline 10 & Leña & Níyá & Serpiente & Nulú Ilacaumé \\
\hline 11 & Bosque & Nacuá & Rana & Naculá \\
\hline 12 & Palo & Ná & Lana & Nyipí numbalatí \\
\hline 13 & Raíz & Nambutih mí & Algodón & Late \\
\hline 14 & Tronco & Numbímu & Lino & No hay \\
\hline 15 & Corteza & Ndúa & Comida & Namutá \\
\hline 16 & Rama & Ndílamú & Crudo & Llupá \\
\hline 17 & Oja & Nímá & Cozer & Unyí \\
\hline 18 & Puerta & Napihamu & Cerbeza & No hay \\
\hline 19 & Ogar & No hay & Vino & Nambam \\
\hline 20 & Ciudad & Nambihihna & Azeite & $\begin{array}{l}\text { Lo mismo en } \\
\text { lengua }\end{array}$ \\
\hline 21 & Villa & Nambihihna & Tierra & Nambulá \\
\hline
\end{tabular}

Fuente: AGI, Documentos Escogidos 1_N165_40_0211. 
Cuadro 9. Vocabulario castellano-chiapaneca, 1789. Hoja 0212

\begin{tabular}{|c|c|c|c|c|}
\hline & Castellano & Chapaneca (sic) & Castellano & Chapaneca (sic) \\
\hline 1 & Seguir & Míláu & Obeja & Humbalatí ñahá \\
\hline 2 & Medida & Nuhunamu & Cabra & Tenchu \\
\hline 3 & Cuchillo & Cuchilu & Cuerno & Nambúmá \\
\hline 4 & Caldero & Natinbucuyá & Caballo & Númbá \\
\hline 5 & Trigo en género & Nama catíla & Camello & No hay \\
\hline 6 & Centeno & No hay & Asno & Burro en lengua \\
\hline 7 & Cerdo & Nuhu & Casa & Nángu \\
\hline 8 & Perro & Numbí & Choza & Nánguñúmú \\
\hline 9 & Gato & Chítu & Tienda & Como en Castilla \\
\hline 10 & León & Nambúma & Como & Cate (advervio) \\
\hline 11 & Oso & No hay & Donde & Ndene? \\
\hline 12 & Lobo & Sacayuyacumé. que & Que & Te \\
\hline 13 & Zorra & No hay & Con quién & Pane mungás \\
\hline 14 & Liebre & No hay & Vajo & Cupúmu \\
\hline 15 & Señor & Chemé & Sin & Tu \\
\hline 16 & Siervo & Numbiumu & Numero & Numahatu \\
\hline 17 & Rey & Rey. I. tiquimusica me & Mesa & Como en Castilla \\
\hline 18 & Ley & Numumbutay mu & Banco & Nambucutá \\
\hline 19 & Leer & Upátu & Cubo & No hay \\
\hline 20 & Principio & Nupuhúmunbatih mi & Navío & Nahuarí acumé \\
\hline 21 & Sí & Cahí & Esquife & Nahuarí acumé \\
\hline
\end{tabular}

Fuente: AGI, Documentos Escogidos 1_N165_40_0212. 
Cuadro 10. Vocabulario castellano-chiapaneca, 1789. Hoja 0213

\begin{tabular}{|c|c|c|c|c|}
\hline & Castellano & Chapaneca (sic) & Castellano & Chapaneca (sic) \\
\hline 1 & Pájaro & Nurí & Coche & No hay \\
\hline 2 & Gorrión & Nurimanyí & Acarrear & Ypumbúta \\
\hline 3 & Golondrina & Nánchurí & Yr en coche & No hay \\
\hline 4 & Pluma & Larí & Construir & Lucite hému nbasé \\
\hline 5 & Huevo & Nbuá & Bestido & Numahaguá mu \\
\hline 6 & Nido & Namandí & Pellizas & No hay \\
\hline 7 & Pastor & Nuumbutahmí & Media & Meria \\
\hline 8 & Arar & Nbiahamu gnuarado & Sapato & Como en Castilla \\
\hline 9 & Arado & $\begin{array}{l}\text { No hay, y se dice como } \\
\text { en Castilla }\end{array}$ & Gorro & Námungúchuma \\
\hline 10 & Carrera & \begin{tabular}{|l} 
Se dice como en \\
Castilla
\end{tabular} & Faja & Como en Castilla \\
\hline 11 & Cosecha & Namahé & Seda & Como en Castilla \\
\hline 12 & Limite & Numucumu & Ciento & Haumíché \\
\hline 13 & Guardia & Numbumámé & Príncipe & Como en Castilla \\
\hline 14 & Yugo & No hay & En & $\mathrm{Mu}$ \\
\hline 15 & Escrivir & Umbaá & Uno & Tiche \\
\hline 16 & Fin & Ypupucú & Ahora & Cateta \\
\hline 17 & No & Úútí & Despues & Llaputí \\
\hline 18 & Manteca & Mandeca & Lejos & Acitú \\
\hline 19 & Pan & Núca catíla & Alla & Tihiñá \\
\hline 20 & Dinero & Tumí & Oy & Llaá \\
\hline 21 & Ladrón & Tipusitía & He aquí & La áta \\
\hline
\end{tabular}

Fuente: AGI, Documentos Escogidos 1_N165_40_0213. 
Cuadro 11. Vocabulario castellano-chiapaneca, 1789. Hoja 0214

\begin{tabular}{|c|c|c|c|c|}
\hline & Castellano & Chapaneca (sic) & Castellano & Chapaneca (sic) \\
\hline 1 & Guerra & No hay & Dos & Húmihí \\
\hline 2 & Soldado & Soldado & Cuatro & Ahuá mihí \\
\hline 3 & Contienda & Ypítátí & Seis & Hambá mihí \\
\hline 4 & Batería & No hay & Ocho & Hahu mihí \\
\hline 5 & Arnez & No hay & Diez & Hénda \\
\hline 6 & Casco & No hay & Veinte & Ahue \\
\hline 7 & Escudo & No hay & Antes & Sícahí \\
\hline 8 & Lanza & Lo mismo en lengua & Cerca & Sindáhamu \\
\hline 9 & Miseria & Napupuí & Aquí & Hin de \\
\hline 10 & Victoria & Nambílaymu & Ayer & Llarihí \\
\hline 11 & Amigo & Nacami mu & Mañana & Mahí \\
\hline 12 & Enemigo & Nampusimey & $\mathrm{O}$ (participio) & Uné \\
\hline 13 & Miel & Nambápu & Tres & Hemihí \\
\hline 14 & Quando & Ndicu mú & Cinco & Haú mihí \\
\hline 15 & Quién & Pane? & Siete & Hendimihí \\
\hline 16 & Con qué & Pe ngáu & Nueve & Helí mihí \\
\hline 17 & Sobre & Muhú & Once & Hénda mundíche \\
\hline 18 & Ternera & Lo mismo en lengua & Treinta & Ahué múnda \\
\hline \multirow[t]{2}{*}{19} & Nuebo & Narímba & Artes & Numusicaýmu \\
\hline & & & Llober & $\begin{array}{l}\text { Rií. con una r, como en ma-rido } \\
\text { en Castilla }\end{array}$ \\
\hline
\end{tabular}

Fuente: AGI, Documentos Escogidos 1_N165_40_0214. 